\title{
UNIQUENESS IN THE LEFT DIVISION OF ORDER TYPES
}

\section{SEYMOUR GINSBURG}

In [7] Sierpiński gave several sufficiency conditions for the uniqueness of left and of right division of order types. Davis, in $[2 ; 3]$, generalized these results for division on the right. The purpose of this note is to consider division on the left.

The following results are needed to prove Theorem 1.

Lemma 1. Let $M$ be a simply ordered set which has the fixed point property. ${ }^{1}$ If $\alpha$ and $\beta$ are any two order types such that $\alpha|M|=\beta|M|$, then $a \equiv \beta$.

The proof of Lemma 1 is to be found in [5].

Lemma 2. Let $H=\{n \mid n<\omega\} \cup\left\{\omega, \omega^{*}, \omega^{*}+\omega\right\}$, and let $\mu$ be any order type such that $\mu<\mu 2 .^{2}$ If $\gamma$ and $\delta$ are any two elements of $H$ such that $\mu \gamma \equiv \mu \delta$, then $\gamma=\delta$.

Proof. In [4] it was shown that $\mu s<\mu t$ for any two positive integers $s$ and $t$ for which $s<t$. Hence the lemma is true if $\gamma$ and $\delta$ are each integers. Since, for $s$ finite, $\mu s<\mu(s+1) \leqq \mu \omega \leqq \mu\left(\omega^{*}+\omega\right)$ and $\mu s<\mu(s+1) \leqq \mu \omega^{*} \leqq \mu\left(\omega^{*}+\omega\right)$, it follows that the lemma is true if one of the elements $\gamma$ or $\delta$ is finite, and the other is not. Suppose that $\mu \omega$ and $\mu \omega^{*}$ are demonstrated to be incomparable order types. ${ }^{2}$ This will imply that $\mu \omega<\mu\left(\omega^{*}+\omega\right)$ and that $\mu \omega^{*}<\mu\left(\omega^{*}+\omega\right)$. Consequently the lemma will be true in all cases. In order to see that $\mu \omega$ and $\mu \omega^{*}$ are incomparable order types let

$$
A=A_{0} \cup A_{1} \cup \ldots \cup A_{n} \cup \ldots \quad(n<\omega)
$$

and

$$
B=\cdots \cup B_{n} \cup \cdots \cup B_{1} \cup B_{0} \quad(n<\omega)
$$

be two ordered sums, ${ }^{3}$ where $\left|A_{i}\right|=\left|B_{j}\right|=\mu$ for each $i$ and $j$. The order

Received by the editors April 20, 1954.

${ }^{1}$ A simply ordered set $E$ is said to have the fixed point property if, for each similarity transformation $f$ of $E$ into $E$, an element $p_{f}$ can be found so that $f\left(p_{f}\right)=p_{f}$.

2 Let $A$ and $B$ be two simply ordered sets. By $|A|<|B|$ is meant that there exists a similarity transformation of $A$ into $B$, but no similarity transformation of $B$ into $A$. By $|A| \equiv|B|$ is meant that there exists a similarity transformation of $A$ into $B$, and a similarity transformation of $B$ into $A .|A|$ and $|B|$ are incomparable order types if there is no similarity transformation of $A$ into $B$ and no similarity transformation of $B$ into $A$.

' Let $E$ be a nonempty simply ordered set. Let $\left\{A_{\mathrm{o}} \mid e \in E\right\}$ be a family of pairwise disjoint sets. The set $B=\bigcup_{e} \in A_{e}$ is called an ordered sum if it satisfies the following two properties for each pair of elements $a_{e}$ and $a_{e^{\prime}}$ of $B$. (1) If $e<e^{\prime}$ then $a_{c}<a_{a^{\prime}}$. (2) If $e=e^{\prime}$ and $a_{\bullet}<a_{e^{\prime}}$ in $A_{e}$, then $a_{\bullet}<a_{e^{\prime}}$ in $B$. 
type of $A$ is $\mu \omega$ and that of $B$ is $\mu \omega^{*}$. Suppose that $\mu \omega$ and $\mu \omega^{*}$ are comparable order types, say $\mu \omega \leqq \mu \omega^{*}$. Thus there exists a similarity transformation $f$ of $A$ into $B$. Let $p$ be an element of $A_{0}$. 'The element $f(p)$ is in one of the sets $B_{i}$, say $B_{m}$. As $f$ is a similarity transformation of $A$ into $B$, for each element $x$ in the set $C=\bigcup_{n \geqq 1} A_{n}, f(x)>f(p)$. This, combined with $f(p)$ being an element of $B_{m}$, implies that $f(x)$ is in the set $D=U_{i \leqq m} B_{m}$, i.e., $f(C) \subseteq D$. Therefore $\mu \omega=|C| \leqq \mu(m+1)$. This is a contradiction. Consequently no such similarity transformation $f$ can exist. Therefore $\mu \omega \leqq \mu \omega^{*}$ is false. In an entirely analogous manner we see that $\mu \omega^{*} \leqq \mu \omega$ is also false. Hence $\mu \omega$ and $\mu \omega^{*}$ must be incomparable order types. Q.E.D.

Let $E$ be a simply ordered set and $p$ an element of $E$. Denote by $F_{p}$ the set of those elements $x$ in $E$ which have the property that there are only a finite number (possibly none) of elements of $E$ between $x$ and $a$. The set $F_{p}$ shall be called a component of $E$.

The components of a set $E$ have the following two properties:

$\left.{ }^{*}\right)$ If $F_{p} \cap F_{q}$ is nonempty, then $F_{p}=F_{q}$.

$\left.{ }^{* *}\right)$ The order type of each component is either $n$, where $n$ is a positive integer, $\omega, \omega^{*}$, or $\omega^{*}+\omega$.

The next lemma is stated as Theorem 2 in [7].

Lemma 3. If $\alpha$ and $\beta$ are any two order types for which $\alpha n=\beta n$, where $n$ is some positive integer, then $\alpha=\beta$.

We now prove our main result.

THEOREM 1. Let $M$ be a simply ordered set with the fixed point property. Let $\alpha$ be any order type such that $\alpha<\alpha 2$. If $\beta$ is any order type such that $\alpha|M|=\beta|M|$, then $\alpha=\beta$.

Proof. Let $A$ and $B$ be two simply ordered sets such that $|A|=\alpha$ and $|B|=\beta$. Let $P=A \times M$ and $Q=B \times M,{ }^{4}$ and $f$ be a similarity transformation of $P$ onto $Q$. For each element $m$ in $M$ let $F_{m}$ be the component of $M$ which contains $m$. Denote by $a_{0}$ a definite element of $A$.

If the order type of each component of $M$ were to be transfinite, i.e., $\omega, \omega^{*}$, or $\omega^{*}+\omega$, then the set $M$ evidently could not have the fixed point property. Therefore one of the components of $M$ must be finite. Let $p$ be the first element of this component and let $f\left(a_{0}, p\right)$ $=\left(b_{0}, q\right)$. Suppose that for some element $(a, m)$ of $A \times F_{p}$, the element $f(a, m)$ is not in $B \times F_{q}$. For each element $(c, z)$ of $A \times F_{p}$ such that

L Let $R$ and $S$ be any two simply ordered sets. By $R \times S$ is meant the cartesian product of the sets $R$ and $S$, i.e., the set $\{(r, s) \mid r \in R, s \in S\}$, the elements being ordered by last differences. 
$\left(a_{0}, p\right)<(c, z)$ consider the element $f(c, z)=(d, u)$. Now

$$
\left(b_{0}, q\right)=f\left(a_{0}, p\right)<f(c, z)=(d, u) .
$$

Suppose that $(d, u)$ is not an element of the set $B \times F_{q}$. Then the set $L=\{w \mid q<w<u, w \in M\}$ must be infinite. For if $L$ were to be finite, then $u$ would be an element of $F_{q}$, whence $(d, u)$ would be in $B \times F_{q}$. Since $z$ is in $F_{p}$ the set $\{x \mid p \leqq x \leqq z, x \in M\}$ has only a finite number of elements, say $r$. Therefore the order type of the set

$$
J=\left\{v \mid\left(a_{0}, p\right)<v<(c, z), v \in P\right\}
$$

is $\leqq|A| r$. Since $L$ is infinite, it contains $r+1$ elements. By Lemma 1 , $\alpha \equiv \beta$. Since $\alpha<\alpha 2$, it follows that $\beta<\beta 2$. By Lemma 2 , it follows that $\beta r<\beta(r+1)$. Thus $\beta r<\beta(r+1) \leqq|B||L|$. Since $f$ is a similarity transformation of $P$ onto $Q$,

$$
f(J)=\left\{y \mid\left(b_{0}, q\right)<y<(d, u), y \in B \times M\right\} .
$$

Hence $B \times L$ is a subset of $f(J)$. Therefore $f^{*}(B \times L)^{5}$ is a subset of $J$. Thus

$$
|B \times L|=\left|f^{*}(B \times L)\right| \leqq|J| \leqq|A| r \equiv|B| r<|B|(r+1) \text {, }
$$

which is a contradiction. Consequently $f(c, z)$ must be an element of $B \times F_{q}$. In a similar manner we see that for each element $(c, z)$ of $A \times F_{p}$ such that $(c, z)<\left(a_{0}, p\right)$, the element $f(c, z)$ is in $B \times F_{q}$. We conclude that $f\left(A \times F_{p}\right)$ is a subset of $B \times F_{q}$.

By considering the function $f^{*}$ we see that $f^{*}\left(B \times F_{q}\right)$ is a subset of $A \times F_{p}$. Thus $B \times F_{q}$ is a subset of $f\left(A \times F_{p}\right)$. Therefore $f\left(A \times F_{p}\right)$ $=B \times F_{q}$. Thus $\left|A \times F_{p}\right|=\left|B \times F_{q}\right| \equiv|A|\left|F_{q}\right|$. By Lemma 2, $\left|F_{p}\right|=\left|F_{q}\right|$. Thus $|A||F|_{p}=|B||F|_{p}$. Since $\left|F_{p}\right|$ is finite, on applying Lemma 3 we obtain $|A|=|B|$. Q.E.D.

If, in Theorem 1, one replaces "the fixed point property" by "a finite component," then the conclusion is no longer valid. For example, let $|M|=\eta, \alpha=1$, and $\beta=\eta$. Each point of $M$ is a component of $M$, and $\alpha|M|=\eta=\beta \eta$. An inspection of the demonstration of Theorem 1 reveals that the proof breaks down at the point where Lemma 1 is applied to obtain $\alpha \equiv \beta$. The reason is that Lemma 1 is no longer valid if "the fixed point property" is replaced by "a finite component."

If the hypothesis on $\alpha$ is removed, then the conclusion may no longer be true. This is so in the case where $|M|=1+\omega^{*}+\omega, \alpha=\eta$, and $\beta=\eta+1$. The condition on $\alpha$ may be relaxed if further conditions on $M$ are assumed. To be specific we have

b By $f^{*}$ is meant the inverse function of $f$. 
THEOREM 2. Let $M$ be a simply ordered set which has a first element, a last element, and the fixed point property. If $\alpha$ and $\beta$ are any two order types for which $\alpha|M|=\beta|M|$ then $\alpha=\beta$.

Proof. Repeat the proof given in [7]. At each place that Sierpiński uses Corollary 2, use Lemma 1 instead.

Now each complete simply ordered set $^{6}$ has both a first and a last element. It is also known that each complete simply ordered set has the fixed point property [6]. Thus we obtain the following known result $[1$, p. 42]:

Corollary. If $A, B$, and $C$ are complete simply ordered sets and $|B||A|=|C||A|$, then $|B|=|C|$.

REMARKs. (1) If $M$ is a well ordered set of order type $\gamma+1$, then $M$ satisfies the hypotheses of Theorem 2 . Consequently Theorem 2 is a generalization of Theorem 2 of [7].

(2) It is natural to inquire as to whether or not the conclusion of Theorem 2 is still true if $M$ is no longer required to have (a) a last element, (b) a first element, and (c) either a first or a last element. In each of the three cases a simple example shows the answer to be in the negative.

(a) Let $|M|=1+\omega^{*}+\omega, \alpha=\eta$, and $\beta=\eta+1$. Then $\alpha|M|=\beta|M|$ $=\eta$.

(b) Let $|M|=\omega^{*}+\omega+1, \alpha=\eta$, and $\beta=1+\eta$. Then $\alpha|M|=\beta|M|$ $=\eta$.

(c) Let $|M|=\omega^{*}+\omega+1+\omega^{*}+\omega, \alpha=\lambda+1$, and $\beta=1+\lambda$. Then $\alpha|M|=\beta|M|=\lambda+\lambda$.

\section{BIBLIOGRAPHY}

1. G. Birkhoff, Lattice theory, rev. ed., New York, 1948.

2. A. C. Davis, Cancellation theorems for products of order types. I, Bull. Amer. Math. Soc. Abstract 58-1-77.

3. - Cancellation theorems for products of order types. II, Bull. Amer. Math. Soc. Abstract 58-4-330.

4. S. Ginsburg, Some remarks on order types and decompositions of sets, Trans. Amer. Math. Soc. vol. 74 (1953) pp. 514-535.

5. - Fixed points of products and ordered sums of simply ordered sets, Proc. Amer. Math. Soc. vol. 5 (1954) pp. 554-565.

6. B. Knaster, Annales de la Société Polonaise de Mathématique vol. 6 (1927) p. 133.

7. W. Sierpinski, Sur la division des types ordinaux, Fund. Math. vol. 35 (1948) pp. 1-12.

UNIVERSity OF MiAMI

- A simply ordered set $E$ is said to be complete if every subset $D$ of $E$ has both a least upper bound and a greatest lower bound in $E$. 